\title{
On the X-Ray Edge Problem in Graphene
}

\author{
Eugene Kogan ${ }^{1,2}$, Moshe Kaveh ${ }^{1}$ \\ ${ }^{1}$ Jack and Pearl Resnick Institute, Department of Physics, Bar-Ilan University, Ramat-Gan, Israel \\ ${ }^{2}$ Max-Planck-Institut fur Physik Komplexer Systeme, Dresden, Germany \\ Email: Eugene.Kogan@biu.ac.il
}

How to cite this paper: Kogan, E. and Kaveh, M. (2018) On the X-Ray Edge Problem in Graphene. Graphene, 7, 1-7. https://doi.org/10.4236/graphene.2018.7100 $\underline{1}$

Received: December 18, 2017

Accepted: January 27, 2018

Published: January 30, 2018

Copyright $\odot 2018$ by authors and Scientific Research Publishing Inc. This work is licensed under the Creative Commons Attribution International License (CC BY 4.0).

http://creativecommons.org/licenses/by/4.0/

\begin{abstract}
We calculate the core-hole spectral density in a pristine graphene, where the density of states of itinerant electrons goes linearly to zero at the Fermi level. We consider explicitly two models of electron-hole interaction. In the unscreened Coulomb interaction model, the spectral density is similar to that in metal (for local interaction). Thus there is no $\delta$-function singularity in the core-hole spectral density. In the local interaction model, the $\delta$-function singularity survives, but the interaction leads to the appearance of the background in the spectral density.
\end{abstract}

\section{Keywords}

Graphene, Dynamical Response

\section{Introduction}

The so-called X-ray edge problem is related to the absorption of high-energy electromagnetic radiation. In the process of $\mathrm{x}$-ray absorption, a core electron absorbs an x-ray photon and is injected from the material. In an insulator, the energy of the ejected electron is unequivocally determined by that of the absorbed photon.

In metal, however, the presence of the conduction electrons makes this process much more interesting. As a result of the core electron ejection, there is a localized change of screened Coulomb potential seen by the conduction electrons. This sudden change of potential leads to transient perturbation of electron gas that is to creation of electron-hole pairs. This process changes the spectral function of a core hole with respect to the case of insulator. It is this spectral function which determines the energy dependence of the cross-section for ejecting photoelectrons from the core state [1]-[6].

Starting from the discovery of graphene, large attention attracts the systems 
which occupy a place intermediate between an insulator and a metal. The gap between the conduction and the valence band in graphene is zero, which allows creation of electron-hole pairs with the energies down to zero, like in a metal. On the other hand, the density of electron states at the Fermi level in the pristine graphene is zero, like in an insulator. So the present paper studies core-hole spectral function in such systems, noticing however, that the results can be applied to any Dirac semimetal.

The X-ray edge problem in graphene [7] was addressed previously [8] [9]. (See also closely connected works, which studied the theory of dynamical spin response for fullerene molecules [10], bilayer graphen [11], topological insulator [12], Kitaev honeycomb model [13] and intercalated graphite [14].) Thus in Ref. [8], both the spectral density of the core hole and the X-ray response function were calculated using the method by Nozieres and de Dominicis [3]. The calculations were done for the local interaction (see below).

In our paper, we calculate the spectral density of the core hole using the method developed by Mahan [1]. For the local interaction, we reproduce the result obtained previously [7]. (We only consider the case of pristine graphene.) For the unscreened Coulomb interaction, we obtain the result which is equivalent to the classical result for the metal with the constant density of states of itinerant electrons but with the local interaction [1]. Thus lower density of the electronhole excitations in graphene in comparison to metal is compensated by singularity of the unscreened Coulomb interaction. As far as the calculation, the X-ray response function is concerned; we only present the general approach to calculation of a one-electron transient Green's function L, leaving the exposition of the results until the next publication.

\section{Spectral Density of the Core Hole}

\subsection{Spectral Density}

The model Hamiltonian is [1]

$$
H=\zeta_{h} d^{\dagger} d+\sum_{k} \zeta_{c}(\boldsymbol{k}) c_{\boldsymbol{k}}^{\dagger} c_{\boldsymbol{k}}+\frac{1}{V} \sum_{\boldsymbol{k} k^{\prime}} V\left(\boldsymbol{k} \boldsymbol{k}^{\prime}\right) c_{\boldsymbol{k}}^{\dagger} c_{\boldsymbol{k}^{\prime}} d^{\dagger} d,
$$

where $d^{\dagger}, d$ are the hole creation and annihilation operators, $c^{\dagger}, c$ are the conduction (valence) creation and annihilation operators.

The hole Green's function is determined as

$$
G_{h}(t)=-i\left\langle T d(t) d^{\dagger}(0)\right\rangle=-i \Theta(t)\left\langle\mathrm{e}^{i H t} d \mathrm{e}^{-i H t} d^{\dagger}\right\rangle \equiv-i \Theta(t) \mathrm{e}^{-i \zeta_{h} t} \rho(t) .
$$

The spectral density is

$$
A_{h}(\Omega)=\int_{-\infty}^{\infty} \frac{\mathrm{d} t}{2 \pi} \mathrm{e}^{i\left(\omega-\zeta_{h}\right) t} \rho(t) .
$$

In the absence of interaction between the core hole and the itinerant electrons

$$
A_{h}(\Omega)=\delta\left(\Omega-\zeta_{h}\right) .
$$

One of the ways to find the Green's function in the presence of interaction is the 
linked cluster expansion

$$
\rho(t)=\left\langle T \exp \left[-i \int_{0}^{t} \mathrm{~d} t_{1} V\left(t_{1}\right)\right]\right\rangle=\exp \left[\sum_{l=1}^{\infty} F_{l}(t)\right]
$$

where

$$
F_{l}(t)=\frac{(-1)^{l}}{l} \int_{0}^{t} \mathrm{~d} t_{1} \cdots \int_{0}^{t} \mathrm{~d} t_{l}\left\langle T V\left(t_{1}\right) \cdots V\left(t_{l}\right)\right\rangle_{\text {connected }}
$$

The first term in the series $\sum_{l=1}^{\infty} F_{l}(t)$ just renormalizes the core level energy. Asuming the electron-hole interaction is weak, we can restrict ourselves by the second term [1]

$$
F_{2}(t)=\frac{1}{V^{2}} \sum_{\boldsymbol{k}_{1} \boldsymbol{k}_{2}}\left|V\left(\boldsymbol{k}_{1} \boldsymbol{k}_{2}\right)\right|^{2}\left[\frac{i t n_{1}}{\zeta_{1}-\zeta_{2}}-\frac{n_{1}\left(1-n_{2}\right)}{\left(\zeta_{1}-\zeta_{2}\right)^{2}}\left(1-\mathrm{e}^{i t\left(\zeta_{1}-\zeta_{2}\right)}\right)\right] .
$$

Using assumption $V\left(\boldsymbol{k}_{1} \boldsymbol{k}_{2}\right)=V\left(\boldsymbol{k}_{1}-\boldsymbol{k}_{2}\right)$ and again ignoring the shift of the hole energy due to the electron-hole interaction, described by the first term in brackets in Equation (7), one obtains

$$
F_{2}(t)=-\int_{0}^{\infty} \frac{\mathrm{d} u}{u^{2}} R_{e}(u)\left(1-\mathrm{e}^{-i u t}\right),
$$

where

$$
R_{e}(u)=-\frac{2}{\pi V} \sum_{\boldsymbol{q}}|V(\boldsymbol{q})|^{2} \operatorname{Im}\left[P^{(1)}(\boldsymbol{q}, u)\right]
$$

and

$$
-\operatorname{Im}\left[P^{(1)}(\boldsymbol{q}, u)\right]=\frac{2 \pi}{V} \sum_{\boldsymbol{k}} n_{F}\left(\zeta_{\boldsymbol{k}}\right)\left[1-n_{F}\left(\zeta_{\boldsymbol{k}+\boldsymbol{q}}\right)\right] \delta\left(u+\zeta_{\boldsymbol{k}}-\zeta_{\boldsymbol{k}+\boldsymbol{q}}\right) .
$$

is the imaginary part of the polarization operator (in a single-bubble approximation). Here and further on we take into account spin and valley degeneracy. For the monolayer graphene [3] [4] [5]

$$
P^{(1)}(\boldsymbol{q}, u)=\frac{q^{2}}{4 \sqrt{v_{F}^{2} q^{2}-u^{2}}} .
$$

\subsection{Local Interaction}

Let the interaction of graphene electrons with the core hole is local $V(\boldsymbol{q})=V=$ const. We obtain

$$
R_{e}(u)=\frac{V^{2}}{4 \pi^{2}} \int_{0}^{u / v_{F}} \frac{q^{3} \mathrm{~d} q}{\sqrt{u^{2}-v_{F}^{2} q^{2}}}=\frac{V^{2} u^{3}}{6 \pi^{2} v_{F}^{4}}
$$

Hence

$$
F_{2}=-\frac{V^{2}}{6 \pi^{2} v_{F}^{4}} \int_{0}^{\xi_{0}}\left(1-\mathrm{e}^{-i u t}\right) u \mathrm{~d} u .
$$

The upper limit of $u\left(\xi_{0}\right)$ can be taken as the band width. Actually, Equations ((9) and (10)) can be joined together to give a simple formula [15] 


$$
\begin{aligned}
R_{e}(u) & =4 V^{2} \int_{\zeta(\boldsymbol{k}>0} \frac{\mathrm{d}^{2} \boldsymbol{k}}{(2 \pi)^{2}} \int_{\zeta\left(\boldsymbol{k}^{\prime}<0\right.} \frac{\mathrm{d}^{2} \boldsymbol{k}^{\prime}}{(2 \pi)^{2}} \delta\left(u-\zeta(\boldsymbol{k})+\zeta\left(\boldsymbol{k}^{\prime}\right)\right) \\
& =\frac{V^{2}}{4} \int_{0}^{\infty} \rho(\epsilon) \mathrm{d} \epsilon \int_{-\infty}^{0} \rho\left(\epsilon^{\prime}\right) \mathrm{d} \epsilon^{\prime} \delta\left(u-\epsilon+\epsilon^{\prime}\right)
\end{aligned}
$$

(where the second equation is correct for a pristine graphene). For monolayer graphene with the dispersion law $\epsilon(\boldsymbol{k})= \pm v_{F} k$ the density is

$$
\rho(\epsilon)=\frac{2|\epsilon|}{\pi v_{F}^{2}} \text {. }
$$

Thus we recover the final result of Equation (12).

The influence of the electron-hole interaction on the core-hole spectral density in graphene for local interaction is much weaker than in the case of Coulomb interaction. In particular, $F_{2}$ defined by Equation (13) converges to the same constant for $t \rightarrow \pm \infty$. Hence $\delta$-function singularity of the spectral density, which we had in the absence of the electron-hole scattering survives in the pristine graphene. However, the interaction gives a background in addition to this singularity, which we'll study in details. For the case of monolayer graphene the background up to a numerical factor can be presented as

$$
A_{b . g}(\Omega)=\int_{-\infty}^{\infty} \frac{\mathrm{d} t}{2 \pi} \mathrm{e}^{\left(\Omega-\zeta_{h}\right) t}\left(\mathrm{e}^{\frac{V^{2}}{6 v_{F}^{4}} \int_{0}^{\xi_{0}} \mathrm{e}^{-i u t} u \mathrm{~d} u}-1\right) .
$$

Calculating integral with respect to $\mathrm{d} u$ we obtain

$$
A_{b . g}(\tilde{\Omega})=\int_{-\infty}^{\infty} \frac{\mathrm{d} t}{2 \pi} \mathrm{e}^{i \tilde{\Omega} t}\left[\mathrm{e}^{g\left[i \mathrm{e}^{\left.-i t / t+\left(\mathrm{e}^{-i t}-1\right) / t^{2}\right]}-1\right],}\right.
$$

where

$$
g=\frac{V^{2} \xi_{0}^{2}}{6 v_{F}^{4}}
$$

Recalling that we assumed from the beginning that $g$ is small, we can expand the exponent in Equation (17). In lowest order approximation we obtain

$$
A_{b . g}(\tilde{\Omega})=g \int_{-\infty}^{\infty} \frac{\mathrm{d} t}{2 \pi} \mathrm{e}^{i \tilde{\Omega} t}\left[\frac{i \mathrm{e}^{-i t}}{t}+\frac{\mathrm{e}^{-i t}-1}{t^{2}}\right]=g \tilde{\Omega} \theta(\tilde{\Omega}) \theta(1-\tilde{\Omega}) .
$$

The result of Equation (19) coincides with that obtained previously [7].

\subsection{Unscreened Coulomb Interaction}

Now consider the case of unscreened Coulomb interaction

$$
V(\boldsymbol{q})=\frac{2 \pi e^{2}}{q}
$$

Substituting imaginary part of Equation (11) and Equation (20) into Equation (9) we obtain

$$
R_{e}(u)=e^{4} \int_{0}^{u / v_{F}} \frac{q \mathrm{~d} q}{\sqrt{u^{2}-v_{F}^{2} q^{2}}}=\frac{e^{4} u}{v_{F}^{2}}
$$


Hence

$$
F_{2}=-g \int_{0}^{\xi_{0}}\left(1-\mathrm{e}^{-i u t}\right) \frac{\mathrm{d} u}{u}
$$

where $g=e^{4} / v_{F}^{2}$. The upper limit of $u$ is taken as the band width $\xi_{0}$. It is interesting that for a monolayer graphene with Coulomb interaction that we obtain the result coinciding with that for a metal with the local interaction $V(\boldsymbol{q})=V=$ const [3], the only difference is that for a metal $g=2\left(N_{F} V\right)^{2}$. Like in the case of metal, $F_{2}$ defined by Equation (22) logarithmically diverges for $t \rightarrow \pm \infty$. This leads to disappearance of the $\delta$-function singularity in the spectral density.

$$
A_{h}(\tilde{\Omega})=\int_{-\infty}^{\infty} \frac{\mathrm{d} t}{2 \pi} \mathrm{e}^{i \tilde{\Omega} t-g \int_{0}^{1}\left(1-\mathrm{e}^{-i u t}\right) \frac{\mathrm{d} u}{u}},
$$

which is equivalent to the classical result for the metal with the constant density of states of itinerant electrons but with the local interaction [1]

$$
A_{h}(\tilde{\Omega})=2 \theta(\tilde{\Omega}) \sin (\pi g) \Gamma(1-g) \frac{\mathrm{e}^{-\tilde{\Omega}}}{\tilde{\Omega}^{1-g}},
$$

where $\tilde{\Omega}=\left(\Omega-\zeta_{h}\right) / \xi_{0}$. Thus lower density of the electron-hole excitations in graphene in comparison to metal is compensated by singularity of the unscreened Coulomb interaction.

\section{Absorption of X-Rays by Core Level to Conduction Band Transition}

The net transition rate in the photoemission experiment in the time representation is expressed as the product of two factors: a one-electron transient Green's function, and the deep-level Green's function $G_{h}$ [3]. In this Section we formulate analog of the Nozieres-de Dominicis equation for the former for the case when the density of states of itinerant electrons goes linearly to zero at the Fermi level.

A one-body transient Green's function $L_{\boldsymbol{k} \boldsymbol{k}^{\prime}}$ is given by

$$
L_{k k^{\prime}}\left(t-t^{\prime}\right)=-\varphi_{k k^{\prime}}\left(t^{\prime}, t ; t, t^{\prime}\right),
$$

and one electron Green's function (calculated in the presence of transient potential) $\varphi$ is defined by equation

$$
\varphi_{\boldsymbol{k} \boldsymbol{k}^{\prime}}\left(\tau, \tau^{\prime} ; t, t^{\prime}\right)=G_{\boldsymbol{k} \boldsymbol{k}^{\prime}}\left(\tau-\tau^{\prime}\right)-i \int_{t}^{t^{\prime}} \mathrm{d} \tau^{\prime \prime} \sum_{\boldsymbol{q} \boldsymbol{q}^{\prime}} G_{\boldsymbol{k} \boldsymbol{q}^{\prime}}\left(\tau-\tau^{\prime \prime}\right) V_{\boldsymbol{q} \boldsymbol{q}^{\prime}} \varphi_{\boldsymbol{q}^{\prime} \boldsymbol{k}^{\prime}}\left(\tau^{\prime \prime}, \tau^{\prime} ; t, t^{\prime}\right),
$$

where

$$
G_{k k^{\prime}}(t)= \begin{cases}\delta_{k k^{\prime}} \mathrm{e}^{-i \epsilon_{\boldsymbol{k}} t} \theta(t) & \text { if } \epsilon_{\boldsymbol{k}}>0 \\ -\delta_{\boldsymbol{k} \boldsymbol{k}^{\prime}} \mathrm{e}^{-i \epsilon_{\boldsymbol{k}} t} \theta(-t) & \text { if } \epsilon_{\boldsymbol{k}}<0\end{cases}
$$

Consider the case of local interaction and assume that electron-hole can be taken as [3]

$$
V_{\boldsymbol{q} \boldsymbol{q}^{\prime}}=-V u(\boldsymbol{q}) u\left(\boldsymbol{q}^{\prime}\right),
$$


where $u$ is a cut-off function. In this case the moments are decoupled, and we can write down closed equation for the quantity

$$
\varphi=\sum_{\boldsymbol{k} \boldsymbol{k}^{\prime}} \varphi_{\boldsymbol{k} \boldsymbol{k}^{\prime}}
$$

Retaining only the "active" variable one obtains [3]

$$
\varphi(\tau)=G\left(\tau-\tau^{\prime}\right)+i V \int_{t}^{t^{\prime}} \mathrm{d} \tau^{\prime \prime} G\left(\tau-\tau^{\prime \prime}\right) \varphi\left(\tau^{\prime \prime}\right) .
$$

The Green's function is

$$
G(t)= \begin{cases}\int_{0}^{\infty} \rho(\epsilon) u^{2}(\epsilon) \mathrm{e}^{-i \epsilon t} \mathrm{~d} \epsilon & (t>0) \\ -\int_{-\infty}^{0} \rho(\epsilon) u^{2}(\epsilon) \mathrm{e}^{-i \epsilon t} \mathrm{~d} \epsilon & (t<0)\end{cases}
$$

Taking the cut-off function as

$$
u(\epsilon)=\mathrm{e}^{-|\epsilon| / \xi},
$$

one obtains

$$
G(t)=\frac{2}{\pi v_{F}^{2}} \frac{1}{(i|t|+1 / \xi)^{2}} .
$$

Equation (30) for $t=0$ and $t^{\prime} \rightarrow \infty$

$$
\varphi(\tau)=G\left(\tau-\tau^{\prime}\right)+i V \int_{0}^{\infty} \mathrm{d} \tau^{\prime \prime} G\left(\tau-\tau^{\prime \prime}\right) \varphi\left(\tau^{\prime \prime}\right),
$$

After being Fourier transformed becomes a Wiener-Hopf equation

$$
\varphi(\omega)=G(\omega) \mathrm{e}^{i \omega \tau^{\prime}}+i V G(\omega) \Phi_{+}(\omega) .
$$

The functions $\Phi_{+}(\omega)$ and $\Phi_{-}(\omega)$, analytic in the upper and lower half planes respectively, are defined as follows

$$
\Phi_{ \pm}(\omega)= \pm \frac{1}{2 \pi i} \int_{-\infty}^{+\infty} \frac{\varphi\left(\omega^{\prime}\right) \mathrm{d} \omega^{\prime}}{\omega^{\prime}-\omega \mp i \delta} .
$$

Using the decomposition

$$
\varphi(\omega)=\Phi_{+}(\omega)+\Phi_{-}(\omega)
$$

we rewrite Equation (35) as

$$
[1-i V G(\omega)] \Phi_{+}(\omega)+\Phi_{-}(\omega)=G(\omega) \mathrm{e}^{i \omega \tau^{\prime}} .
$$

Solution of Equation (38) with the Green' function (33) will be a subject of further research.

\section{Conclusions}

We calculated the spectral density of the core hole for pristine graphene for two models of electron-hole interaction: unscreened Coulomb and local interaction.

The calculations are performed using the method developed by Mahan. We also formulate analog of Nozieres-de Dominices equation for calculating the net transition rate in the photoemission experiment in graphene. 


\section{Acknowledgements}

E.K. cordially thanks Max-Planck-Institut fur Physik Komplexer Systeme for the hospitality extended to him during his visit there.

\section{References}

[1] Mahan, G. (1967) Exitons in Metals: Infinite Hole Mass. Physical Review, 163, 612617.

[2] Anderson, P.W. (1967) Infrared Catastrophe in Fermi Gases with Local Scattering Potentials. Physical Review Letters, 18, 1049-1051. http://doi.org/10.1103/Phys.Rev.Lett.18.1049

[3] Nozieres, P. and de Dominicis, C.T. (1969) Singularities in the X-Ray Absorption and Emission of Metals. III. One-Body Theory Exact Solution. Physical Review, 178, 1097-1107.

[4] Schotte, K.D. and de Schotte, U. (1969) Tomonaga's Model and the Threshold Singularity of X-Ray Spectra of Metals. Physical Review, 182, 479-482.

[5] Doniach, S. and Sondheimer, E.H. (1998) Green's Functions for Solid State Physicists. Imperial College Press, London. https://doi.org/10.1142/p067

[6] Mahan, G.D. (2000) Many-Particle Physics. 3rd Edition, Kluver Academics/Plenum Publishers, New York. https://doi.org/10.1007/978-1-4757-5714-9

[7] Castro Neto, A.H., Guinea, F., Peres, N.M.R., Novoselov, K.S. and Geim, A.K. (2009) The Electronic Properties of Graphene. Reviews of Modern Physics, 81, 109162. http://doi.org/10.1103/RevModPhys.81.109

[8] Yang, S.-R.E. and Lee, H.C. (2007) X-Ray Edge Problem of Graphene. Phys. Rev. B, 76, 245411-1-245411-7.

[9] Gonzalez, J., Guinea, F. and Vozmediano, M.A.H. (1993) Electrostatic Screening in Fullerene Molecules. Modern Physics Letters B, 7, 1593-1599. http://doi.org/10.1142/S0217984993001612

[10] Roder, G., Tkachov, G. and Hentschel, M. (2011) Photoabsorption Spectra and the X-Ray Edge Problem in Graphene. Europhysics Letters, 94, 67002-p1-67002-p6. https://doi.org/10.1209/0295-5075/94/67002

[11] Lee, H.C. (2010) X-Ray Edge Singularity of Bilayer Graphene. Phys. Rev. B, 82, 153410-1-153410-10.

[12] Lee, H.C. (2010) Theoretical Study of X-Ray Absorption of Three-Dimensional Topological Insulator $\mathrm{Bi}_{2} \mathrm{Se}_{3}$. Phys. Rev. B, 83, 193107-1-193107-4.

[13] Knolle, J., Kovrizhin, D.L., Chalker, J.T. and Moessner, R. (2015) Dynamics of Fractionalization in Quantum Spin Liquids. Phys. Rev. B, 92, 115127-p1-115127-p20.

[14] Shung, K.V.K. (1986) Dielectric Function and Plasmon Structure of Stage-1 Intercalated Graphite. Phys. Rev. B, 34, 979-993.

[15] Gogolin, A.O., Nersesyan, A.A. and Tsvelik, A.M. (1998) Bosonization and Strongly Correlated Systems. Cambridge University Press, Cambridge. 